\title{
Intervenções escolares para redução da obesidade infantil: uma revisão sistemática
}

\author{
School-based interventions for reducing childhood obesity: a systematic review
}

\author{
Julia Andradea, Luísa Carolina Bischoffa, Leticia Bonetto ${ }^{a}$, Caroline Pieta Dias ${ }^{\mathrm{b}}$, Cristian Roncadac $^{\mathrm{c}}$ \\ a Acadêmicos de Biomedicina, Faculdade da Serra Gaúcha (FSG). \\ b Educadora Física. Doutora em Ciências do Movimento Humano, Universidade Federal do Rio Grande do Sul. Professora da FSG. \\ c Educador Físico. Doutor em Pediatria e Saúde da Criança, Pontifícia Universidade Católica do Rio Grande do Sul. Professor da FSG.
}

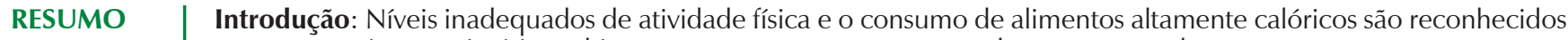
como mecanismos primários subjacentes ao aumento no excesso de peso corporal.

Objetivo: Avaliar as principais intervenções voltadas à redução da obesidade infantil no âmbito escolar.

Materiais e Métodos: Uma revisão sistemática foi realizada para identificar estudos de intervenção aplicados à obesidade infantil escolar nos últimos 10 anos. Foi aplicada uma lógica de pesquisa nas bases de dados PubMed, Scielo e ScienceDirect. Para seleção dos artigos, dois pesquisadores analisaram os mesmos considerando os critérios de inclusão e exclusão de forma independente. Quando necessário, um terceiro revisor também analisou os artigos. Resultados: Dos 16 artigos analisados pela sistemática, a grande maioria utiliza a atividade física $(\mathrm{n}=13)$ ou a educação nutricional $(n=12)$ como foco intervencional nos cuidados da obesidade infantil escolar. Boa parte dos estudos foca seus cuidados aos escolares $(n=11)$, possuindo um número reduzido de estudos que estendem a educação aos pais $(n=6)$ e professores $(n=3)$. Também são poucos os estudos que utilizam grupo controle para comparar a qualidade das intervenções $(n=6)$. Contudo, os achados demonstram que a intervenção primária focada em educação sobre hábitos de vida saudáveis traz benefícios ao público escolar.

Conclusão: Existem evidências que a melhor forma de controle da obesidade infantil baseia-se na intervenção primária, focada na prevenção e proteção à saúde por meio de programas educativos, tendo o ambiente escolar e o convívio familiar como os principais norteadores deste desfecho.

Palavras-chaves: obesidade; qualidade de vida; criança.

Introduction: Poor nutrition and insufficient physical activity, due to the consumption of high-calorie foods are recognized as primary mechanisms underlying the increase in excess body weight.

Objective: To evaluate the main interventions aimed at reducing childhood obesity in schools.

Materials and Methods: A systematic review was conducted to identify studies of interventions applied to school childhood obesity in the last 10 years. A rationale for the search was applied on PubMed, SciELO and ScienceDirect databases. For selection of articles, two researchers independently analyzed the criteria of inclusion and exclusion. When necessary, a third reviewer also analyzed the studies. Results: Of the 16 articles selected by the systematic search, most uses physical activity $(n=13)$ or nutrition education $(n=12)$ as the main intervention for the care of school childhood obesity. Much of the research focuses its care for the children ( $n=11)$, having a small number of studies that extend education to parents $(n=6)$ and teachers $(n=3)$. Also, there are few studies using control groups to compare the quality of interventions $(n=6)$. However, the findings demonstrate that primary interventions focused on education about healthy living may benefit the school public.

Conclusion: There is evidence that the best way to control childhood obesity is based on primary interventions focused on prevention and health protection, through educational programs, being the school environment and the family considered as the main drivers of this outcome.

Keywords: obesity; quality of life; child. 


\section{INTRODUÇÃO}

A obesidade infantil tem alcançado proporções epidêmicas ${ }^{1}$, sendo considerada uma doença crônica, chamando a atenção de pesquisadores em saúde pela elevada prevalência nas ultimas décadas ${ }^{2}$. Esta doença ganha cada vez mais espaço no campo de pesquisas, reportagens e demais meios de comunicação ${ }^{3}$. No entanto, ainda faltam ferramentas e métodos realmente eficazes para o manejo desta doença ${ }^{2}$.

Em países de primeiro mundo, como no caso dos Estados Unidos, Canadá, Austrália, entre outros países da Europa, ao longo das últimas quatro décadas, as taxas de obesidade infantil têm aumentado de forma preocupante ${ }^{4}$, tendo como principais causas os fatores ambientais, em conjunto das mudanças de hábito de vida, aumentando os níveis de sedentarismos, bem como da inadequada ingesta nutricional, baseada em insumos industralizados ${ }^{1}$.

O aumento do acúmulo de gordura corporal acaba por prejudicar a saúde das crianças, afetando negativamente as condições físicas e metabólicas, contribuindo para a resistência à insulina e a inflamação crônica ${ }^{3}$. Com isso, o excesso de peso infantil pode ocasionar na redução do bem estar físico, bem como da autoestima, contribuindo para o impacto de doenças na vida adulta como hipertensão, diabetes tipo II, doenças cardiovasculares e os vários tipos de cânceres, que resultam na diminuição da qualidade de vida, além do impacto financeiro a saúde pública ${ }^{5}$.

Existe uma crescente necessidade para o desenvolvimento de intervenções para reduzir a prevalência da obesidade infantil em fase escolar, sendo as mesmas baseadas em evidências de que a obesidade está relacionada com o aumento no consumo de alimentos calóricos na dieta e um estilo de vida cada vez mais sedentário ${ }^{6}$. Tais intervenções devem centrar-se na mudança deste comportamento, buscando orientar não só o público infantil, mas também seus familiares que, de certa forma, possuem influência direta na mudança de hábitos de vida, seja no fator sedentarismo bem como na alimentação não saudável ${ }^{7}$.

As escolas têm sido um cenário popular e importante para implementação de intervenções, uma vez que oferecem contato contínuo com as crianças ${ }^{8}$. No entanto, apesar das vantagens aparentes de tratar a obesidade infantil no ambiente escolar, tem se observado uma relativa falta de eficácia nas intervenções e recursos escassos para intervenções no ambiente escolar ${ }^{9}$.

A maioria das pesquisas realizadas em ambiente escolar tem sido focadas na oferta da educação nutricional, ${ }^{10}$ diminuição do tempo gasto em frente as telas (televisão, videogame e computadores), redução do comportamento sedentário com distribuição de panfletos e palestras educativas bem como programas de atividade física, e na modificação do cardápio oferecido pelas cantinas escolares ${ }^{11}$. No entanto, a maioria dos estudos são realizados sob a premissa de que as mudanças na dieta e na atividade física serão alcançadas se forem realizados na idade adulta, prevenindo a obesidade em anos posteriores ${ }^{12}$. Contudo, pensando em uma atualização baseada em evidências dos estudos de intervenção aplicados nos últimos 10 anos, a presente revisão tem como objetivo avaliar as principais intervenções no âmbito escolar focadas na obesidade infantil.

\section{MATERIAIS E MÉTODOS}

Para o desenvolvimento deste trabalho se fez uso de uma lógica de pesquisa para identificar estudos nos últimos 10 anos que aplicaram intervenções voltadas à redução da obesidade infantil em escolas.

\section{Critérios de inclusão}

Para inclusão nesta revisão sistemática, os artigos obrigatoriamente deveriam tratar-se de estudos de intervenção. Além disso, deveriam descrever detalhadamente o protocolo de intervenção, como por exemplo: tipo de intervenção, local de aplicação, tempo, entre outros. Artigos sem essas informações foram excluídos, assim como os artigos que não estavam disponíveis na integra para leitura.

\section{Estratégia de busca}

Como estratégia de busca foi adotada a lógica baseada em descritores específicos (em língua inglesa), vinculadas aos operadores booleanos (AND), sendo aplicada da seguinte forma: (Obesity AND School AND Intervention). As buscas foram aplicadas nas bases de dados PubMed, Scielo e ScienceDirect, no período de setembro de 2014. Para evitar a inclusão excessiva de artigos, foram delimitadas as buscas nos últimos 10 anos e nos seguintes campos: título (Title), palavras-chave (Keywords) e resumo (Abstract). Desta forma, os três descritores obrigatoriamente deveriam constar em pelos menos um dos três campos de busca.

Além destes, não foram adicionados filtros de limitação, como por exemplo: língua do artigo ou público alvo. As exportações dos artigos foram feitas nas extensões: Medline, Ris e Bibtex, e as importações foram feitas por meio do software específico para elaboração de revisões sistemática StArt (State of the Art through Systematic Review) ${ }^{13}$, servindo como apoio na identificação dos artigos duplicados, excluídos e incluídos. As análises foram feitas separadamente por três pesquisadores e analisada por outros dois revisores.

Como critério de relevância na seleção, foram adotadas três etapas para inclusão e exclusão dos artigos, sendo: a) artigos selecionados pelos três pesquisadores foram incluídos automaticamente; b) artigos não selecionados pelos três pesquisadores foram excluídos automaticamente; 
c) artigos selecionados por dois dos três pesquisadores, foram analisadas pelos dois revisores. No caso de discordância dos revisores, os artigos foram excluídos e no caso de concordância foram incluídos. Mediante tais etapas, foi feita a verificação das consistências dos artigos incluídos, em conjunto pelos cinco pesquisadores.

\section{Qualidade metodológica}

A qualidade metodológica dos estudos primários foi avaliada pelo sistema GRADE que foi proposto pelo grupo Grades of Recommendation, Assessment, Developmentand Evaluation ${ }^{14}$. Além disso, após a seleção dos estudos, foi aplicado um checklist contendo perguntas (Tabela 3) para verificar o nível de significância e inferência científica dos mesmos.

\section{RESULTADOS}

No total, foram encontrados 945 artigos (PubMed=644; ScienceDirect $=245$; Scielo=56), por meio das buscas eletrônicas. Inicialmente 104 artigos foram excluídos em virtude de duplicidade e 758 por não se tratarem de estudos de intervenção. Assim, foram pré-selecionados 83 artigos, dos quais 67 foram excluídos por não se enquadrarem nos critérios de inclusão. Assim, nesta revisão foram incluídos para análise 16 estudos clínicos (Figura 1).

Os dados referentes aos artigos incluídos nesta revisão são apresentados nas Tabelas 1 e 2. Na Tabela 1, são abordados resultados de caracterização geral dos estudos, como autores, ano de publicação, total de participantes, período de início das coletas, além do país de origem dos estudos. Já na Tabela 2, são apresentados os tipos de intervenções, bem como o período de vigência do protocolo, público alvo e os tipos de grupos de comparação.

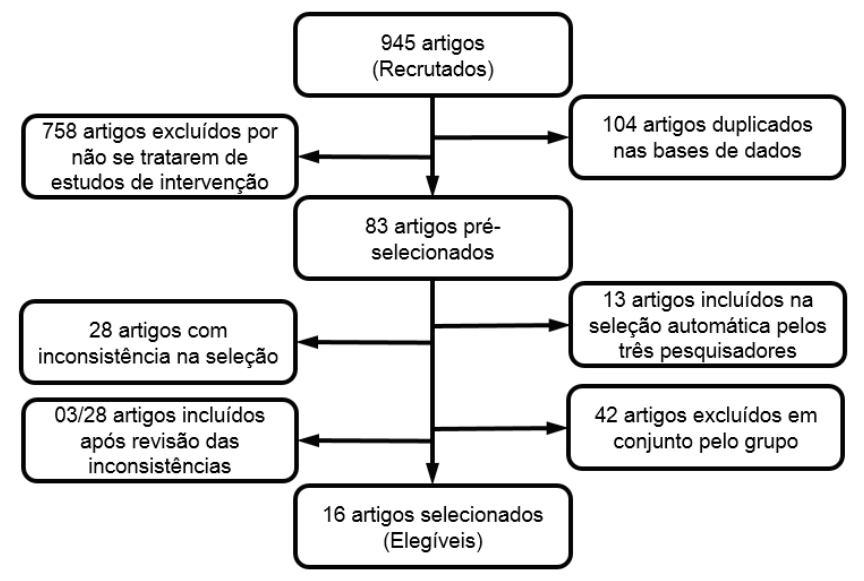

Figura 1. Fluxograma da seleção dos estudos.

Tabela 1 - Identificação geral dos estudos elegíveis na sistemática.

\begin{tabular}{|c|c|c|c|c|}
\hline Autor & $\begin{array}{c}\text { Ano } \\
\text { (publicação) }\end{array}$ & $\stackrel{\mathbf{n}}{\text { (participantes) }}$ & $\begin{array}{l}\text { Período } \\
\text { (coleta) }\end{array}$ & $\begin{array}{l}\text { Local } \\
\text { (País) }\end{array}$ \\
\hline Baker et al. ${ }^{15}$ & 2013 & 78 & 2008 & Inglaterra \\
\hline Binkinet al. ${ }^{16}$ & 2008 & 161 & 2007 & Inglaterra \\
\hline Blüheret al. ${ }^{17}$ & 2014 & 142 & NC & USA \\
\hline Costa et al. ${ }^{18}$ & 2014 & 2.155 & 2006 & Brasil \\
\hline Gabriele et al. ${ }^{19}$ & 2010 & 773 & 2007 & USA \\
\hline Garcia et al. ${ }^{20}$ & 2013 & 1.224 & 1999 & México \\
\hline Jaballaset al. ${ }^{21}$ & 2011 & 992 & 2009 & USA \\
\hline Klakket al. ${ }^{22}$ & 2013 & 632 & 2011 & Dinamarca \\
\hline Lee et al..$^{23}$ & 2013 & 562 & NC & USA \\
\hline Marques et al..$^{24}$ & 2013 & 1.396 & $\mathrm{NC}$ & Brasil \\
\hline Nataleet al..$^{25}$ & 2013 & 1.105 & 2010 & USA \\
\hline Pallanet al. ${ }^{26}$ & 2013 & 88 & 2008 & Irã \\
\hline Pérez et al. ${ }^{27}$ & 2010 & 1.283 & $\mathrm{NC}$ & Espanha \\
\hline Robinson et al. ${ }^{28}$ & 2013 & 261 & 2011 & USA \\
\hline Vargas et al..$^{29}$ & 2011 & 331 & 2005 & Brasil \\
\hline Vasquez et al..$^{30}$ & 2013 & 120 & NC & Chile \\
\hline
\end{tabular}

NC: Não consta.

Tabela 2 - Síntese dos tipos, período e grupos avaliados nas intervenções.

\begin{tabular}{|c|c|c|c|c|}
\hline Autor & Intervenção & $\begin{array}{l}\text { Período de } \\
\text { intervenção }\end{array}$ & Público Alvo & Grupos avaliados \\
\hline Baker et al. ${ }^{15}$ & Atividade Física e Nutricional & 24 anos & Escolares e familiares & Grupo intervenção \\
\hline Binkin et al. ${ }^{16}$ & Atividade Física e Nutricional & 12 meses & Pais e cuidadores & Grupo intervenção \\
\hline Blüher et al. ${ }^{17}$ & Atividade Física & 12 meses & Escolares e seus irmãos & Grupo intervenção \\
\hline Costa et al. ${ }^{18}$ & Atividade Física e Nutricional & 10 meses & Escolares & Grupo intervenção \\
\hline Gabriele et al. ${ }^{19}$ & $\begin{array}{l}\text { WebSite sobre educação } \\
\text { para redução de peso }\end{array}$ & 24 meses & Escolares & Grupo intervenção \\
\hline Garcia et al. ${ }^{20}$ & Atividade Física e Nutricional & 36 meses & Escolares & Grupo intervenção e Grupo controle \\
\hline Jaballas et al. ${ }^{21}$ & Atividade Física e Nutricional & 24 meses & Pais e cuidadores & Grupo intervenção \\
\hline Klakk et al. ${ }^{22}$ & Atividade Física & 24 meses & Escolares & Grupo intervenção e Grupo controle \\
\hline Lee et al. ${ }^{23}$ & Nutricional & 8-10 semanas & Professores & Grupo intervenção \\
\hline Marques et al. ${ }^{24}$ & Avaliação nutricional & NC & Escolares & Grupo intervenção \\
\hline Natale et al. ${ }^{25}$ & Atividade Física e Nutricional & 36 meses & Pais, cuidadores e professores & Grupo intervenção e Grupo controle \\
\hline Pallan et al. ${ }^{26}$ & Atividade Física e Nutricional & NC & Pais, cuidadores e professores & Grupo intervenção \\
\hline Pérez et al. ${ }^{27}$ & Atividade Física e Nutricional & NC & Escolares e familiares & Grupo intervenção \\
\hline Robinson et al. ${ }^{28}$ & Atividade Física & 24 meses & Escolares e familiares & Grupo Intervenção e Grupo Educação (Controle) \\
\hline Vargas et al. ${ }^{29}$ & Atividade Física e Nutricional & 4 meses & Escolares & Grupo intervenção e Grupo controle \\
\hline Vasquez et al. ${ }^{30}$ & $\begin{array}{l}\text { Atividade Física, Nutricional } \\
\text { e Psicológica }\end{array}$ & 4 meses & Escolares & Grupo intervenção e Grupo controle \\
\hline
\end{tabular}


Tabela 3 - Avaliação metodológica segundo os critérios GRADE.

\begin{tabular}{|c|c|c|c|c|c|c|c|c|c|c|c|c|c|c|c|c|}
\hline Parâmetros avaliados & 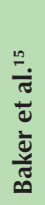 & 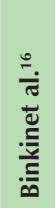 & 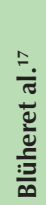 & $\begin{array}{l}\frac{\infty}{\pi} \\
\frac{\pi}{0} \\
\frac{\pi}{0} \\
0\end{array}$ & 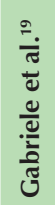 & 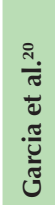 & 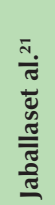 & 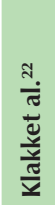 & 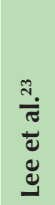 & 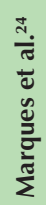 & 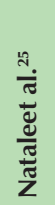 & 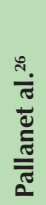 & 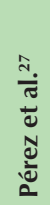 & 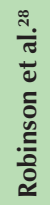 & 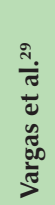 & 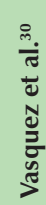 \\
\hline 1. Os desfechos apresentados são relevantes cientificamente? & $\mathrm{S}$ & $\mathrm{S}$ & $\mathrm{S}$ & $\mathrm{S}$ & $\mathrm{S}$ & $\mathrm{S}$ & $\mathrm{S}$ & $\mathrm{S}$ & $\mathrm{S}$ & $\mathrm{S}$ & $\mathrm{S}$ & $\mathrm{S}$ & $\mathrm{S}$ & $\mathrm{S}$ & $\mathrm{S}$ & $\mathrm{S}$ \\
\hline $\begin{array}{l}\text { 2. A metodologia é descrita de forma clara e condiz com o } \\
\text { desfecho principal? }\end{array}$ & $\mathrm{N}$ & $\mathrm{S}$ & $\mathrm{S}$ & $\mathrm{S}$ & $\mathrm{N}$ & $\mathrm{S}$ & $\mathrm{S}$ & $\mathrm{N}$ & $\mathrm{N}$ & $\mathrm{S}$ & $\mathrm{N}$ & $\mathrm{S}$ & $\mathrm{S}$ & $\mathrm{S}$ & $\mathrm{S}$ & $\mathrm{N}$ \\
\hline $\begin{array}{l}\text { 3. Como desfecho principal, utilizam intervenção aplicada à } \\
\text { obesidade infantil? }\end{array}$ & $\mathrm{S}$ & $\mathrm{S}$ & $\mathrm{S}$ & $\mathrm{S}$ & $\mathrm{S}$ & $\mathrm{S}$ & $\mathrm{S}$ & $\mathrm{S}$ & $\mathrm{S}$ & $\mathrm{S}$ & $\mathrm{S}$ & $\mathrm{S}$ & $\mathrm{S}$ & $\mathrm{S}$ & $\mathrm{S}$ & $\mathrm{S}$ \\
\hline 4. Há outra associação com a obesidade infantil? & $\mathrm{S}$ & S & $\mathrm{N}$ & S & $\mathrm{N}$ & S & $\mathrm{S}$ & $\mathrm{S}$ & $\mathrm{N}$ & $\mathrm{N}$ & $\mathrm{N}$ & $\mathrm{N}$ & $\mathrm{N}$ & $\mathrm{S}$ & $\mathrm{N}$ & $\mathrm{N}$ \\
\hline 5. Há um grupo controle para comparar o desfecho principal? & $\mathrm{N}$ & $\mathrm{N}$ & $\mathrm{S}$ & $\mathrm{S}$ & $\mathrm{N}$ & $\mathrm{N}$ & $\mathrm{N}$ & S & $\mathrm{N}$ & $\mathrm{N}$ & $\mathrm{N}$ & $\mathrm{S}$ & $\mathrm{N}$ & $\mathrm{N}$ & S & $\mathrm{N}$ \\
\hline 6. A amostra é estatisticamente significativa? & $\mathrm{S}$ & $\mathrm{S}$ & $\mathrm{S}$ & $\mathrm{S}$ & $\mathrm{S}$ & $\mathrm{S}$ & $\mathrm{S}$ & $\mathrm{S}$ & $\mathrm{S}$ & $\mathrm{S}$ & $\mathrm{S}$ & $\mathrm{S}$ & $\mathrm{S}$ & $\mathrm{S}$ & $\mathrm{S}$ & $\mathrm{S}$ \\
\hline 7. Os autores expressam de forma clara os resultados? & S & $\mathrm{S}$ & $\mathrm{S}$ & $N$ & $\mathrm{~N}$ & $\mathrm{~S}$ & $\mathrm{~S}$ & $\mathrm{~N}$ & $\mathrm{~S}$ & $\mathrm{~S}$ & $\mathrm{~N}$ & $\mathrm{~S}$ & $\mathrm{~S}$ & $\mathrm{~S}$ & $\mathrm{~S}$ & $\mathrm{~S}$ \\
\hline 8. Os resultados vão de encontro com a hipótese? & $\mathrm{S}$ & $\mathrm{S}$ & $\mathrm{S}$ & S & S & $\mathrm{S}$ & $\mathrm{S}$ & $\mathrm{S}$ & $\mathrm{S}$ & $\mathrm{S}$ & $\mathrm{S}$ & $\mathrm{S}$ & $\mathrm{S}$ & $\mathrm{S}$ & $\mathrm{S}$ & $\mathrm{S}$ \\
\hline 9. Os autores expressam as limitações do estudo? & $\mathrm{N}$ & $\mathrm{S}$ & $\mathrm{N}$ & $N$ & $\mathrm{~N}$ & $\mathrm{~N}$ & $\mathrm{~N}$ & $\mathrm{~S}$ & $\mathrm{~N}$ & $\mathrm{~S}$ & $\mathrm{~S}$ & N & $\mathrm{N}$ & $\mathrm{N}$ & $\mathrm{S}$ & $\mathrm{N}$ \\
\hline 10. A conclusão traz alguma inferência científica? & $\mathrm{S}$ & $\mathrm{S}$ & $\mathrm{S}$ & $\mathrm{S}$ & $\mathrm{S}$ & $\mathrm{S}$ & $\mathrm{S}$ & $\mathrm{S}$ & $\mathrm{S}$ & $\mathrm{S}$ & $\mathrm{S}$ & $\mathrm{S}$ & $\mathrm{S}$ & $\mathrm{S}$ & $\mathrm{S}$ & S \\
\hline 11. Os potenciais conflitos de interesse são declarados? & $\mathrm{S}$ & NC & $\mathrm{S}$ & $\mathrm{S}$ & NC & NC & NC & NC & NC & $\mathrm{S}$ & $\mathrm{NC}$ & $\mathrm{S}$ & NC & NC & NC & $\mathrm{NC}$ \\
\hline
\end{tabular}

S: Sim; N: Não; NC: Não consta.

Dos 16 estudos elegíveis na sistemática, apenas três ${ }^{19,23-24}$ não tiveram seus desfechos baseados na atividade física, tendo como foco a educação nutricional (2/3) e o uso de aplicativos website para educação (1/3) na qualidade de vida saudável. Na mesma proporção (12/16) foram os estudos que utilizaram a educação nutricional como um dos desfechos na intervenção e apenas um estudo ${ }^{30}$ utilizou além da atividade física e nutricional a intervenção focada nos cuidados psicológicos de seus participantes. A grande maioria das intervenções foi aplicada por um período igual ou superior a 12 meses (9/16), sendo aplicadas a escolares (11/16), pais ou cuidadores (6/16), familiares $(4 / 16)$ e professores (3/16). Além disso, apenas 6/16 utilizaram grupo controle para comparação com o grupo intervenção.

Após a análise detalhada dos artigos, foi verificado com base nos resultados, que a intervenção escolar auxilia na prevenção da obesidade infantil. Notou-se também que crianças que frequentam cultos religiosos, encontram nele uma forma de apoio para a reversão da obesidade. Em contra partida, um fato que auxilia na ocorrência da doença é o pouco conhecimento dos pais e responsáveis dos fatores predisponentes para a doença.

No período pré-intervenção, as crianças obesas possuíam baixa autoestima. Já, após a intervenção, em função da prática de exercício e da evidenciada redução de peso, as crianças passaram a ter mais disposição, fazendo com que a autoestima melhorasse. A maior parte dos pais envolvidos apresentou maior conhecimento sobre a doença de seus filhos, passando a dar maior atenção e apoio na questão da dieta balanceada e rotina de exercícios físicos.

\section{DISCUSSÃO}

Atualmente a sociedade está vivenciando um mundo repleto de mudanças com foco na facilitação das atividades de vida diária e a falta de tempo para a prática de atividades físicas, bem como para uma alimentação saudável. Mesmo assim, tem sido evidenciado um apelo social e científico sobre a importância da intervenção baseada em hábitos de vida saudáveis, principalmente na saúde infantil, com foco na obesidade. Neste sentido, estudos baseados em evidências sobre a importância da readequação desta nova tendência, são de extrema importância no âmbito da saúde pública, tendo como foco à intervenção primária focada na prevenção, proteção e promoção à saúde do público infantil.

Nas escolas, os estudos mostram que o mais importante e benéfico às crianças obesas é a reeducação alimentar e a prática de exercícios físicos ${ }^{17,20,21,30}$. Alguns artigos trazem a importância do conhecimento da doença, propondo aos responsáveis e aos professores palestras e acompanhamentos ${ }^{15,18,26}$. Já em casa, os métodos não são tão diferentes aos aplicados na escola. Entretanto, em casa é preciso que os pais observem os hábitos dos filhos, incentivando a prática de exercícios físicos, evitando a alimentação junto à televisão ou computador. Alguns estudos demonstram que em casa, a cobrança dois pais pode ser maior e a atenção precisa ser mais intensa do que a dispensada na comunidade escolar ${ }^{15,20,29}$. 
Nos estudos descritos por Blüher et al. ${ }^{17}$, Gabriele et al. ${ }^{19}$, Jaballas et al. ${ }^{21}$, Lee et al..$^{23}$ e Robinson et al. ${ }^{28}$ (Tabela 1), realizados nos Estados Unidos, possuem na sua maioria o desfecho de identificar a eficácia dos métodos de prevenção e redução do número de crianças obesas, com exceção do estudo escrito por Jaballaset al. ${ }^{21}$, que tem como objetivo constatar a percepção dos pais sobre o peso e situação que encontram-se seus filhos em relação a obesidade. Por mais que estes estudos tenham sido realizados no mesmo país, estes possuem diferenças, como por exemplo, o estudo realizado por Blüher et al. ${ }^{17}$, apresentando um período delimitado de um ano. Já no estudo proposto por Robinson et al. ${ }^{28}$ teve como objetivo implementar o método de educação nutricional no ambiente escolar, sem tempo exato de término da intervenção.

Na América Central, Garcia et al. ${ }^{20}$ desenvolveram um estudo randomizado controlado, com objetivo de descrer os resultados de um programa de adoção de estilo de vida saudável, aplicado por três anos, sendo avaliada a redução do índice de massa corporal (IMC) e do consumo de alimentos em escolares de duas comunidades Mexicanas, sendo aplicada a intervenção em 816 escolares, comparando com um grupo controle de 408 escolares. A intervenção foi realizada em escolas primárias e consistiu de educação sobre hábitos saudáveis, modificação de alimentos distribuídos e atividade física. Como resultado no transcorrer dos três anos, houve uma redução significativa no IMC dos escolares (grupo intervenção) em comparação ao grupo controle, além dos escores de redução na ingesta calóricas dos mesmos.

Já na América do Sul, Vasquez et al. ${ }^{30}$ realizaram um estudo para avaliar o impacto de uma intervenção no sistema de ensino, incluindo exercício de força muscular, educação nutricional e aconselhamento aplicados aos escolares obesos. No estudo, aplicaram a intervenção em 120 escolares, como idade de oito a 13 anos, caracterizados como obesos, divididos em dois grupos. No grupo $1(n=60)$ os escolares foram submetidos a um programa de exercícios físicos, educação nutricional e aconselhamento por 12 semanas e o grupo 2 recebeu apenas a intervenção educativa e de apoio psicológico no mesmo período. Na intervenção foram avaliados o IMC, circunferência da cintura, gordura corporal, síndrome metabólica e fatores de risco cardiovasculares. Como resultados, encontraram diferenças significativas entre os grupos na mudança do IMC, circunferência abdominal, gordura corporal, síndrome metabólica, obesidade abdominal, hipertrigliceridemia e hiperglicemia de jejum. Neste estudo, os autores, demonstraram haver um impacto positivo do exercício sobre a força muscular na redução da gordura corporal, síndrome metabólica e fatores de risco cardiovascular, apoiando uso do exercício como um tratamento para a obesidade e suas comorbidades escolares.

No Brasil, assim como nos outros países citados anteriormente, nas últimas décadas a taxa de obesidade infantil vem crescendo sem controle. Com isso, fica evidente a preocupação científica sobre o tema, sendo selecionados três estudos (Vargas et al. ${ }^{29}$, Costa et al. ${ }^{18}$ e Marques et al. ${ }^{24}$ nacionais na presente revisão. Os artigos escritos por Vargas et al. ${ }^{29}$ e Marques et al. ${ }^{24}$ incentivavam a prática de exercícios físicos e uma alimentação saudável. Além disso, o estudo de Vargas et al. ${ }^{29}$ aborda uma intervenção por meio de roteiro de hábitos saudáveis para prevenção da obesidade em alunos de qualquer faixa etária. Já o artigo proposto por Costa et al. ${ }^{18}$ prioriza aplicar seus métodos de intervenção somente com os alunos que estudavam no período noturno, causando desinteresse dos escolares à pratica de atividades física por trabalharem no período diurno. Ainda, no artigo escrito por Marques et al. ${ }^{24}$, não havia programas de atividade física ou de reeducação alimentar, como nos artigos citados anteriormente, sendo aplicada apenas avaliação de medidas de peso, estatura e circunferência da cintura periodicamente. Como conclusão, os autores descreveram que em escolas particulares o índice de obesidade entre os alunos é significativamente maior do que em escolas públicas.

Apesar de todos os artigos selecionados para este estudo, terem o mesmo propósito (aplicar intervenções e entender as causas da obesidade infantil), alguns possuem características bem especificas, tanto no âmbito metodológico quanto no estilo de intervenção, tomando-se como exemplo o artigo escrito por Baker et al. ${ }^{15}$ que acrescentou a participação do seu público em cultos religiosos. Em decorrência disso, recebeu o apoio da religião, para aplicar a intervenção no período extra escolar. No estudo escrito por Binkinet al. ${ }^{16}$, onde aplicou uma intervenção direcionada às mães dos escolares, por meio de questionários, conseguiram estabelecer uma associação entre a falta de conhecimento destas mães e a obesidade de seus filhos.

Dos artigos selecionados para fazerem parte desta sistemática, o que apresentou resultados mais relevante foi o escrito por Lee et al..$^{23}$, onde realizaram uma intervenção focada numa disciplina do ensino médio, intitulada "Educação Nutricional" a qual tinha como objetivo ensinar aos escolares os benefícios de uma vida saudável, comprovando que a implantação deste tipo de disciplina nas escolas, reduz significativamente o índice de obesidade infantil, ocasionando aumento de bons hábitos de estilo de vida, reduzindo a alimentação baseada em "fastfoods".

Por fim, os estudos evidenciam que a melhor forma de controle da obesidade infantil baseia-se na intervenção primária, focada na prevenção e proteção à saúde, por meio 
de programas educativos, de práticas de atividades físicas e de conscientização de hábitos de vida saudáveis, tendo o ambiente escolar e o convívio familiar como os principais norteadores deste desfecho. Acredita-se que ainda possam ser encontrados novos métodos e resultados ainda mais significantes, podendo agregar ainda mais conhecimento científico a esta realidade de obesidade infantil.

\section{REFERÊNCIAS}

1. Collins CA, Champlin SE, Pasch KE, Williams JD. Introduction: Childhood Obesity: media, advertising, community, and advocacy. In: Williams JD, Pasch KE, Collins CA. Advances in Communication Research to Reduce Childhood Obesity. Berlin: Springer; 2013. p. 3-18. http://dx.doi.org/10.1007/978-1-4614-5511-0_1

2. Pate RR, Pratt M, Blair SN, Haskell WL, Macera CA, Bouchard C, Buchner D, Ettinger W, Heath GW, King AC, Kriska A, Leon AS, Marcus BH, Morris J, Paffenbarger-Jr RS, Patrick K, PollockML, Rippe JM, MD; Sallis J, Wilmore JH. Physical activity and public health: a recommendation from the Centers for Disease Control and Prevention and the American College of Sports Medicine. Jama 1995;273(5):402-7. http://dx.doi.org/10.1001/ jama.1995.03520290054029

3. Franks PW, Hanson RL, Knowler WC, Sievers ML, Bennett PH, Looker HC. Childhood obesity, other cardiovascular risk factors, and premature death. New Engl J Med 2010;362(6):485-93. http:// dx.doi.org/10.1056/NEJMoa0904130

4. Chen AY, Kim SE, Houtrow AJ, Newacheck PW. Prevalence of obesity among children with chronic conditions. Obesity 2010;18(1):210-3. http://dx.doi.org/10.1038/oby.2009.185

5. Perez MK, Piedimonte G. Metabolic Asthma: is there a link between obesity, diabetes, and asthma? Immunol Allergy Clin North Am 2014;34(4):777-84. http://dx.doi.org/10.1016/j.iac.2014. 07.002

6. Schmidt MI, Duncan BB, Menezes AM, Monteiro CA, Barreto SM, Chor D, Menezes PR. Chronic non-communicable diseases in Brazil: burden and current challenges. The Lancet 2011;377(9781): 1949-61. http://dx.doi.org/10.1016/S0140-6736(11)60135-9

7. Han JC, Lawlor DA, Kimm S. Childhood obesity. The Lancet 2010;375(9727):1737-48. http://dx.doi.org/10.1016/S01406736(10)60171-7

8. Millimet DL, Tchernis R, Husain M. School nutrition programs and the incidence of childhood obesity. J Hum Res 2010;45(3):640-54. http://dx.doi.org/10.1353/jhr.2010.0021

9. Brophy S, Rees A, Knox G, Baker J, Thomas NE. Child fitness and father's BMI are important factors in childhood obesity: a school based cross-sectional study. PLoS One 2012;7(5):e36597. http:// dx.doi.org/10.1371/journal.pone.0036597

10. Silveira JA, Taddei JA, Guerra PH, Nobre MR. Effectiveness of schoolbased nutrition education interventions to prevent and reduce excessive weight gain in children and adolescents: a systematic review. J Ped 2011;87(5):382-92. http://dx.doi.org/10.2223/ jped.2123

11. Gupta N, Goel K, Shah P, Misra A. Childhood obesity in developing countries: epidemiology, determinants, and prevention. Endocr Rev 2012;33(1):48-70. http://dx.doi.org/10.1210/er.2010-0028
12. Agostoni C, Braegger C, Decsi T, Kolacek S, Koletzko B, Mihatsch W, Moreno LA, Puntis J, Shamir R, Szajewska H, Turck D, Goudoever JV. Role of dietary factors and food habits in the development of childhood obesity: a commentary by the ESPGHAN Committee on Nutrition. J Ped Gastroent Nutr 2011;52(6):662-9. http://dx.doi. org/10.1097/MPG.0b013e3182169253

13. Zamboni A, Di Thommazo A, Hernandes E, Fabbri S. StArt uma ferramenta computacional de apoio à revisão sistemática. In: Congresso Brasileiro de Software (CBSoft'10), Salvador, Brazil; 2010 .

14. Guyatt G, Oxman AD, AkI EA, Kunz R, Vist G, Brozek J, Norris S, Falck-Ytter Y, Glasziou P, DeBeer H, Jaeschke R, Rind D, Meerpohl J, Dahm P, Schünemann HJ. GRADE guidelines: GRADE evidence profiles and summary of findings tables. J Clin Epidemiol 2011; 64(4):383-94. http://dx.doi.org/10.1016/j.jclinepi.2010.04.026

15. Baker G, Maynard M, Rawlins E, Harding S. Addressing complexity of context: an exploratory study of childhood obesity prevention among diverse ethnic groups in schools and places of worship. The Lancet 2013;382:S72. http://dx.doi.org/10.1016/S01406736(13)62497-6

16. Binkin N, Spinelli A, Baglio G, Lamberti A. What is common becomes normal: The effect of obesity prevalence on maternal perception. Nutr Metab Cardiovasc Dis 2013;23(5):410-6. http:// dx.doi.org/10.1016/j.numecd.2011.09.006

17. Blüher S, Petroff D, Wagner A, Warich K, Gausche R, Klemm $\mathrm{T}$, Wagner $\mathrm{H}$, Keller UM. The one year exercise and lifestyle intervention program: effects on anthropometric parameters, cardiometabolic risk factors and glycemic control in childhood obesity. Metabolism 2014;63(3):422-30. http://dx.doi. org/10.1016/j.metabol.2013.11.016

18. Costa FFd, Assis MAAd, González-Chica D, Bernardo C, Barros MVGd, Nahas MV. Effect of school-based intervention on diet in high school students. Rev Bras Cineantr Desemp Hum 2014; 16:36-45.

19. Gabriele JM, Stewart TM, Sample A, Davis AB, Allen R, Martin CK, Newton RL, Williamson DA. Development of an internet-based obesity prevention program for children. J Diabetes Sci Technol 2010;4(3):723-32. http://dx.doi.org/ $10.1177 / 193229681000400328$

20. Alvirde-García U, Rodríguez-Guerrero AJ, Henao-Morán S, Gómez-Pérez FJ, Aguilar-Salinas CA. Resultados de un programa comunitario de intervención en el estilo de vida en ni-os. Sal Públ Méx 2013;55:406-14.

21. Jaballas E, Clark-Ott D, Clasen C, Stolfi A, Urban M. Parents' perceptions of their children's weight, eating habits, and physical activities at home and at school. J Ped Heal Care 2011;25(5): 294-301. http://dx.doi.org/10.1016/j.pedhc.2010.05.003

22. Klakk H, Chinapaw M, Heidemann M, Andersen LB, Wedderkopp $\mathrm{N}$. Effect of four additional physical education lessons on body composition in children aged 8-13 years a prospective study during two school years. BMC Ped 2013;13(1):170. http://dx.doi. org/10.1186/1471-2431-13-170

23. Lee $\mathrm{H}$, Contento IR, Koch P. Using a systematic conceptual model for a process evaluation of a middle school obesity risk-reduction nutrition curriculum intervention. J Nutr Educ Behav 2013; 45(2):126-36. http://dx.doi.org/10.1016/j.jneb.2012.07.002 
24. Ferreira Marques CD, Ribeiro Silva Rde C, Machado ME, Portela de Santana ML, Castro de Andrade Cairo R, Pinto Ede J, Oliveira Reis Maciel L, Rodrigues Silva L. The prevalence of overweight and obesity in adolescents in Bahia, Brazil. Nutr Hosp 2013;28(2):491-6.

25. Natale R, Scott SH, Messiah SE, Schrack MM, Uhlhorn SB, Delamater A. Design and methods for evaluating an early childhood obesity prevention program in the childcare center setting. BMC Public Health 2013;13:78. http://dx.doi.org/10.1186/1471-2458-13-78

26. Pallan M, Mohammadpour-Ahranjani B, Rashidi A, Adab P. Contributors to childhood obesity in Iran: the views of parents and school staff. Public Health 2014;128(1):83-90. http://dx.doi. org/10.1016/j.puhe.2013.10.005

27. Pérez SV, Rodríguez-Martín A, Ruiz JN, Nieto JM, Campoy JL. Hábitos y estilos de vida modificables en ni-os con sobrepeso y obesidad. Nutr Hosp 2010;25(5):823-31.
28. Robinson TN, Matheson DM, Kraemer HC, Wilson DM, Obarzanek E, Thompson NS, Alhassan S, Spencer TR, Haydel F, Fujimoto M, Varady A, Killen JD. A randomized controlled trial of culturally tailored dance and reducing screen time to prevent weight gain in low-income African American girls: Stanford GEMS. Arch Ped Adol Med 2010;164(11):995-1004. http://dx.doi.org/10.1001/ archpediatrics.2010.197

29. Vargas ICS, Sichieri R, Sandre-Pereira G, da Veiga GV. Avaliação de programa de prevenção de obesidade em adolescentes de escolas públicas. Rev Saúde Pública 2011;45(1):59-68. http://dx.doi. org/10.1590/S0034-89102011000100007

30. Vásquez F, Díaz E, Lera L, Meza J, Salas I, Rojas P, Atalah E, Burrows, R. Impacto del ejercicio de fuerza muscular en la prevención secundaria de la obesidad infantil; intervención al interior del sistema escolar. Nutr Hosp 2013;28(2):347-56. 